\title{
Improvement of PVA Gel Properties for Cell Immobilization
}

\author{
Riham Surkatti ${ }^{1,2}$, Muftah H. El-Naas ${ }^{1}$, Mark C.M. Van Loosdrecht ${ }^{2}$, Fatima Al-Naemi ${ }^{3}$, Udeogu \\ Onwusogh $^{4}$ \\ ${ }^{1}$ Gas Processing Center, Qatar University, Doha, Qatar \\ Muftah@qu.edu.qa; Riham.abubakr@qu.edu.qa \\ ${ }^{2}$ Department of Biotechnology, Delft University of Technology \\ NL 2628, BC Delft, The Netherlands \\ M.C.M.vanLoosdrecht@tudelft.nl \\ ${ }^{3}$ Department of Biology, Qatar University, Doha, Qatar \\ f.naemi@qu.edu.qa \\ ${ }^{4}$ Qatar Shell RTC, Doha, Qatar. \\ Udeogu.Onwusogh@shell.com
}

\begin{abstract}
This study investigates the characterization and the ability of polyvinyl alcohol (PVA) gel to be used in the area of wastewater treatment as biomass immobilization matrix. The PVA matrix was prepared through the freezing-thawing cross-linking process under several preparation conditions including freezing-thawing temperature and number of cycles. PVA morphology and pore structure were tested using SEM analysis. In addition, mechanical characterization of the PVA gel was carried out using compression tests. The optimum conditions for the preparation of PVA gel with high pore structure and mechanical strength were determined to be freezing at $-20^{\circ} \mathrm{C}$ and thawing at $+20^{\circ} \mathrm{C}$, and this can be achieved at 4 or 8 iterative freezing-thawing cycles. The prepared PVA gel under these conditions can be utilized for biomass immobilization in the area of biological wastewater treatment.
\end{abstract}

Keywords: Poly vinyl alcohol (PVA) gel, Morphology, Mechanical strength, Immobilization.

\section{Introduction}

PVA is a linear polymer that was traditionally cross-linked by irradiation [1] or bio-functional groups containing chemical agents, such as alginate [2], glutaraldehyde [3] or boric acid [4]. Due to the limitation of these methods, a different physical crosslinking method, which uses an iterative freezing-thawing process on concentrated PVA aqueous solution, has been adopted to produce hydrogel with elastic, rubbery and spongy structure, with good mechanical strength [5]. The selection of polymer type in the preparation of physically cross-linked gel is critical and depends on two factors: the formation of network that sealed large amount of water molecules and strong interaction through the chain in order to form semi-permanent junction in the molecular network [6]. Generally, in the area of biological wastewater treatment, the preparation of an immobilization matrix is carried out using PVA alone or in combination with other polymer, such as chitosan, in which the physical cross-linking takes place by hydrogen bonding interaction obtained through iterative freezing-thawing cycles $[7,8]$. The freezing-thawing technique has been widely applied in the area of wastewater treatment due its biocompatibility and non-toxicity; it also produces a hydrogel that has desirable physical properties and highly porous structure [9]. As a synthetic polymer, PVA is nontoxic, inexpensive, which makes it suitable for microbial immobilization. It also has high chemical and mechanical resistance and easier film forming ability [2,10].The mechanical strength and pores structure of the PVA prepared by freezing-thawing is affected by number of factors including PVA concentration, the number of thermal cycles, thawing rate and thawing temperature.

The objective of this study is to prepare PVA matrices with high porosity and mechanical stability to be used as biomass immobilization carrier. This paper presents the evaluation of the effect of preparation conditions, including freezing thawing temperature and number of thermal cycles on the hydrogel porous structure and mechanical stability. 


\section{Materials and methods}

\subsection{PVA preparation}

PVA matrices were prepared through the freezing-thawing method using analytical grade PVA powder, obtained from $\mathrm{BDH}, \mathrm{UK}$. A homogenous 10 mass\% PVA solution was prepared by mixing $20 \mathrm{~g}$ of PVA powder with $180 \mathrm{ml}$ of distilled water at about $70-80^{\circ} \mathrm{C}$. The PVA powder was added slowly to the hot water with vigorous mixing to make a well mixed, homogenous solution. The mixture was then cooled in the refrigerator, poured into special molds and kept in a freezer for $24 \mathrm{hr}$ before transferring and allowing them to thaw for 6 hours. The freezing-thawing temperature were varied for each prepared matrix. The freezing thawing process was repeated for four cycles to ensure good cross-linking. Another batch of PVA homogenous solutions were prepared at the optimum freezing-thawing temperature based on the above procedure at several freezing-thawing cycles. After preparation, the morphology and mechanical strength were tested for all PVA samples.

\subsection{Porosity and Mechanical properties}

All prepared PVA samples were cut into cubes with nominal dimensions of $16 \mathrm{~mm}$ width, $17 \mathrm{~mm}$ depth and $18 \mathrm{~mm}$ length; they were then freeze-dried for $24 \mathrm{hr}$ before the investigation. The microstructure of the prepared PVA matrices were examined using Scanning Electron Microscopy (SEM), Nova Nano SEM 450, which can provide high quality images with high resolution. Compression tests were performed on the samples using a Universal Material Testing machine MTS of 50 $\mathrm{kN}$ load cell. The compression tests were conducted at room temperature and at a constant overhead speed of $25 \mathrm{~mm} / \mathrm{min}$ until the sample reached ultimate failure (complete compression). The tests were carried out to evaluate the compression properties of the PVA matrices (stress-strain and compression strength).

\section{Results and discussion}

\subsection{Effect of freezing thawing temperature}

In order to obtain PVA matrices with high porosity and mechanical stability, PVA matrices were first prepared at several preparation conditions, including different freezing-thawing temperatures and number of thermal cycles. The effect of the freezing-thawing temperatures on the structure and porosity were tested at several values of Freezing $\left({ }^{\circ} \mathrm{C}\right)$ : Thawing $\left({ }^{\circ} \mathrm{C}\right)$ of $-20:+5,-20:+20,-5:+5$ and $-5:+20$. The prepared PVA gel was analyzed to evaluate the porosity and morphology using SEM. Results showed that at freezing: thawing temperature of $-5^{\circ} \mathrm{C}:+5^{\circ} \mathrm{C}$ and $-5^{\circ} \mathrm{C}:+20^{\circ} \mathrm{C}$, no gelation or cross-linking occurred and the process resulted in a viscous PVA solution without solidification. In contrast, at the other freezing-thawing temperature ranges $\left(-20{ }^{\circ} \mathrm{C}:+5{ }^{\circ} \mathrm{C}\right.$ and $\left.-20{ }^{\circ} \mathrm{C}:+20{ }^{\circ} \mathrm{C}\right)$, the PVA was completely cross-linked and stable. The porous structure of the PVA matrices prepared at freezing temperature of $-20 \mathrm{C}$ and thawing temperatures of $+5^{\circ} \mathrm{C}$ and $+20^{\circ} \mathrm{C}$, were investigated using SEM analysis. As shown in Figure 1, low pore distribution with small pore size is observed for the PVA gels prepared at $\mathrm{F}$ : $\mathrm{T}$ of $-20^{\circ} \mathrm{C}:+5^{\circ} \mathrm{C}$, while dense and larger pore distribution with clear network structure is observed for the gel prepared at $\mathrm{F}$ : $\mathrm{T}$ of $-20^{\circ} \mathrm{C}:+20^{\circ} \mathrm{C}$. Thus, this temperature range $\left(\mathrm{F}: \mathrm{T}\right.$ of $\left.-20^{\circ} \mathrm{C}:+20^{\circ} \mathrm{C}\right)$ is considered to be the best cross-linking condition to prepare a PVA matrix with high porosity. These results may be attributed to the importance of the wide variation between freezing and thawing temperatures to allow the formation of a strong polymeric network within the gel.

(a)

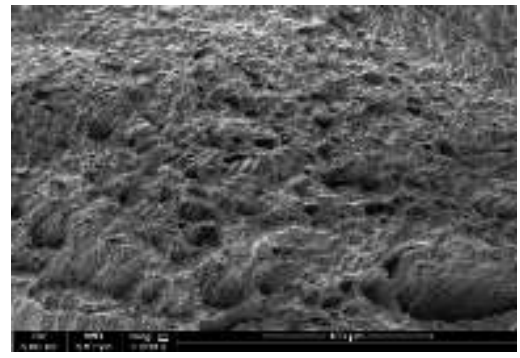

(b)

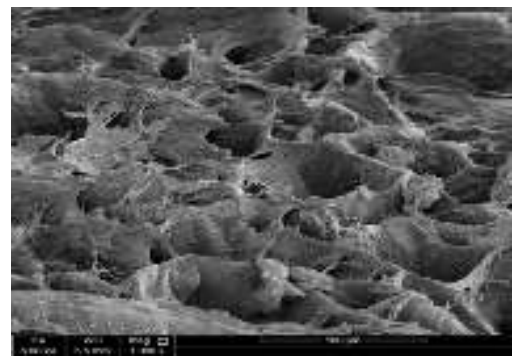

Fig. 1: SEM images of PVA at several freezing-thawing temperature. (a) $\mathrm{F}: \mathrm{T}$ of $-20^{\circ} \mathrm{C}:+5{ }^{\circ} \mathrm{C}$; (b) $\mathrm{F}: \mathrm{T}$ of $-20{ }^{\circ} \mathrm{C}:+20^{\circ} \mathrm{C}$.

The compression test was carried out for cross-linked PVA gel prepared under several freezing-thawing temperatures (Figure 2). Clearly, preparation of the PVA gel at $\mathrm{F}$ : $\mathrm{T}$ of $-20^{\circ} \mathrm{C}:+20^{\circ} \mathrm{C}$ results in the formation of strong and stable gel. The compressive strength increased from 125 to $158 \mathrm{MPa}$ by reducing the freezing temperature from -5 to $-20{ }^{\circ} \mathrm{C}$, resulting 
in a stronger and more elastic gel. The enhancement in the mechanical strength maybe due to the formation of highly crosslinked PVA gel under these conditions.

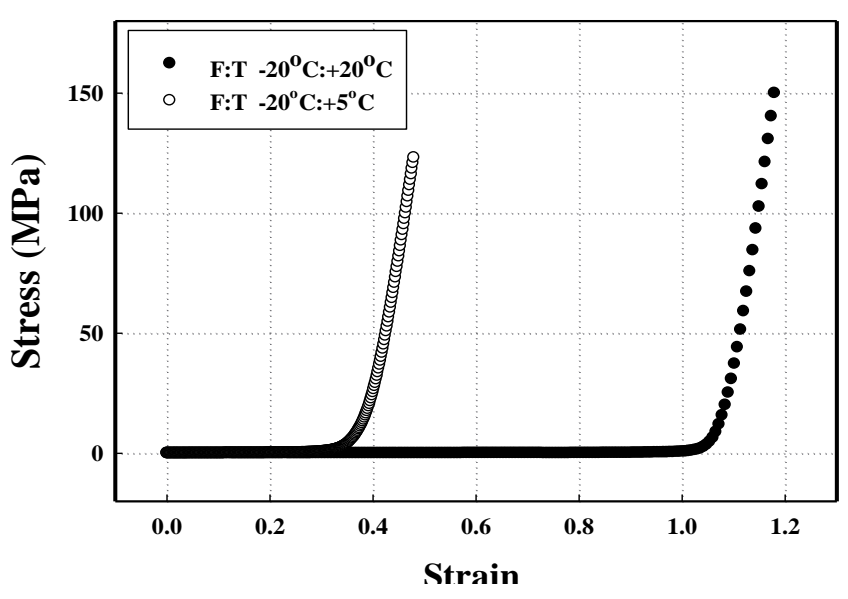

(a)

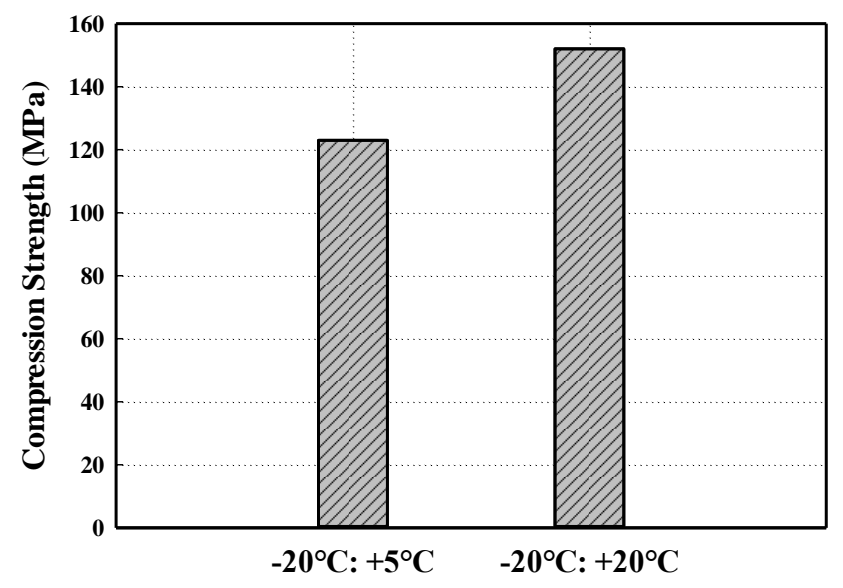

(b)

Fig. 2: stress-strain curve for PVA gel prepared under several freezing-thawing temperatures, (a) stress-strain curve (b) compression strength.

\subsection{Effect of freezing-thawing cycles}

Once the best freezing-thawing temperature range is determined, it is important to examine the effect of the number of cycles on the PVA structure and mechanical strength. PVA gel matrices were prepared at 4 and 8 freezing-thawing cycles, keeping the freezing-thawing temperature at $-20^{\circ} \mathrm{C}:+20^{\circ} \mathrm{C}$. Clearly porosity and pore distribution seem to increase with increasing the number of cycles from 4 and 8 (Figure 3). In addition, fine pores structure with uniform distribution is resulted from the increment in the thermal preparation cycles. It should be mentioned here that increasing the number of thermal cycles is generally increased the size of the initial crystallites or forming new secondary ones [11]. Stauffer and Peppast [12] studied the effect of number of cycles on the PVA gel using several thermal cycle numbers and concluded that higher number of cycles resulted in denser and regular distribution of pores all over the gel.

(a)

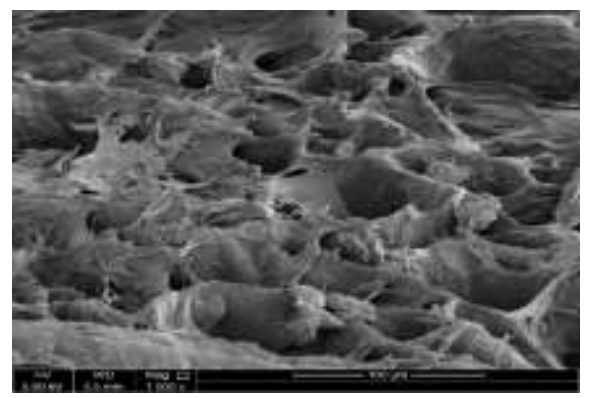

(b)

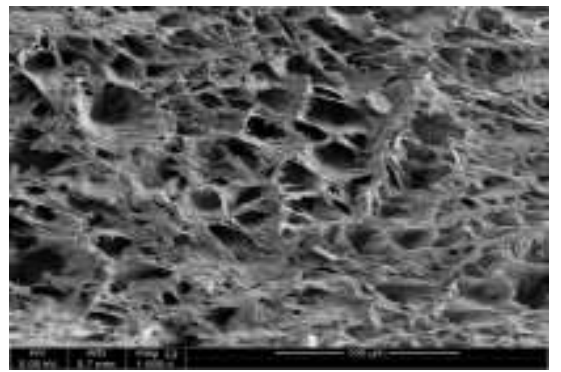

Fig. 3: SEM images of PVA at several freezing-thawing temperature. (a) 4 cycles: (b) 8 cycles. 
The effect of freeze/thaw cycles on the compressive strength of the PVA gel is shown in Figure 4. It can be concluded that the compressive strength of PVA gel increases with the rise of freeze/thaw cycles from 4 to 8 cycles. Furthermore, the influence degree of freeze/thaw cycles on the compressive strength of the PVA gel reduces with the increase of freeze/thaw cycles from 4 to 8, and the compressive strength increased from 159 to $172 \mathrm{MPa}$. The effect of number of cycles on the mechanical strength of PVA gel was tested using Universal Material Testing Machine. Stress-strain curves showed that there are no significant difference on the compression strength for PVA gel prepared at 4 and 8 freezing-thawing cycles (Figure 4 a). The mechanical strength is slightly increased with increasing number of cycles, and higher mechanical strength is achieved at 8 freezing-thawing cycles. Jiang et al. [13] also investigated the effect of freeze/thaw cycles on the PVA gel strength and concluded the mechanical strength improved by increasing number of cycles from 3 to 7 cycles. These results may be attributed to the uniform distribution of the pores through the PVA gel. A previous study by Pazos et al [14] reported similar findings and indicated that increasing the number of freezing-thawing cycles results in a stronger PVA gel structure due to increasing the number of intermolecular hydrogen bonding, which is believed to enhance the aggregation of the molecular chains. However, the cost associated with carrying out 8 cycles and the effect of extended periods of freezing on bacterial cells may overweigh the limited incremental improvements in mechanical strength. Therefore, 4 is considered to be a sufficient number of cycles for preparing a high quality PVA matrix with good porous structure and mechanical strength.

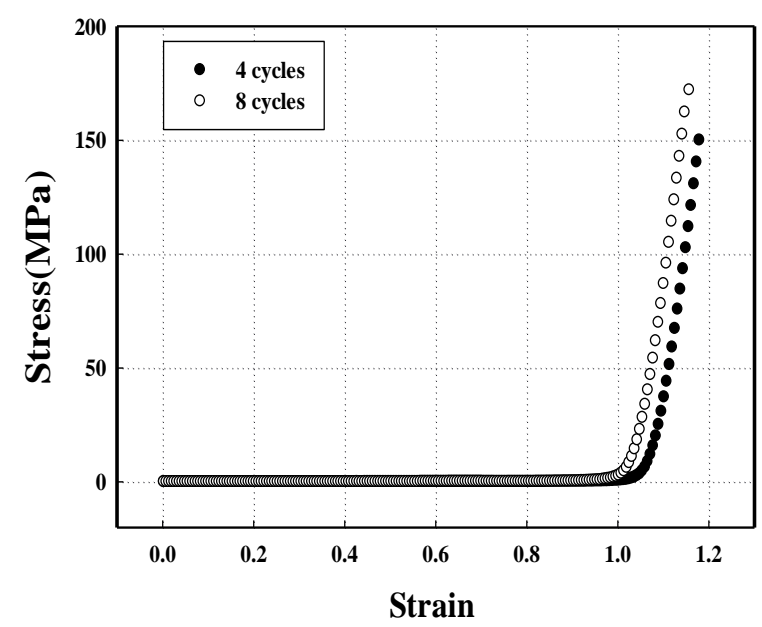

(a)

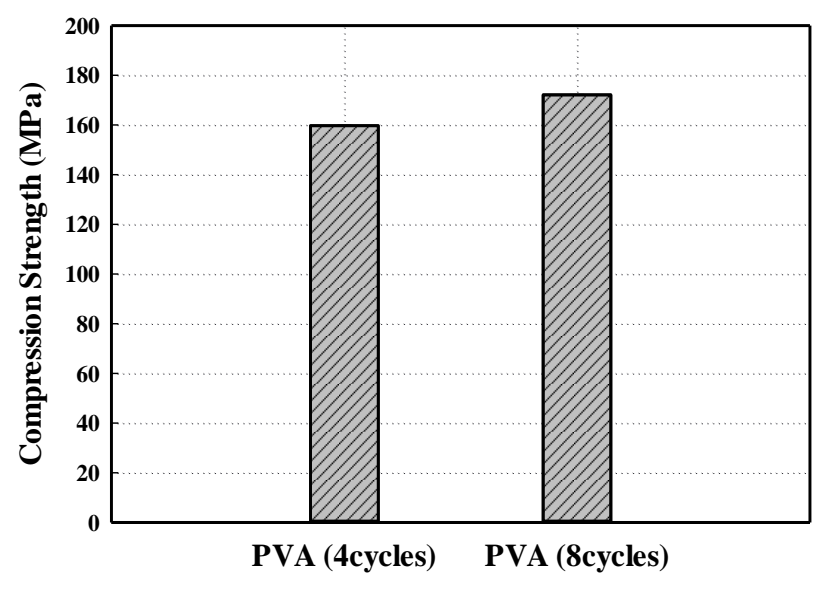

(b)

Fig. 4: Mechanical strength of PVA gel prepared at several F-T cycles; (a) stress-strain curve: (b) compression strength.

\section{Conclusions}

Porous structure and mechanical strength of PVA matrices seemed to depend heavily on preparation conditions such as freezing-thawing temperature and the number of freezing-thawing cycles. A high quality PVA matrices were prepared in the study at a freezing-thawing $(\mathrm{F}: \mathrm{T})$ of $-20 \mathrm{C}:+20 \mathrm{C}$, using four cycles. Maintaining these appropriate preparation conditions will results in a PVA gel that is suitable for cell immobilization. This study will be extended to include the addition of nanoparticles as additive to PVA matrices to improve the mechanical properties and durability of the PVA as an effective immobilization carrier

\section{Acknowledgments}

The authors would like to acknowledge the support of Qatar National Research Fund (a member of Qatar Foundation) through Grant \# NPRP 100129170278. The findings achieved herein are solely the responsibility of the authors.

\section{References}

[1] M. T. Razzak, S. P. Dewi, H. Lely, and E. Taty, "The characterization of dressing component materials and radiation formation of PVA \pm PVP hydrogel," vol. 55, pp. 153-165, 1999.

[2] E. A. Kamoun, E. S. Kenawy, T. M. Tamer, M. A. El-meligy, and M. S. Mohy, "Poly ( vinyl alcohol ) - 
alginate physically crosslinked hydrogel membranes for wound dressing applications : Characterization and bio-evaluation," Arab. J. Chem., vol. 8, no. 1, pp. 38-47, 2015.

[3] C. Yeom and K. Lee, "Pervaporation separation of water-acetic acid mixtures through poly ( vinyl alcohol ) membranes crosslinked with glutaraldehyde," vol. 109, pp. 257-265, 1996.

[4] T. Miyazaki, Y. Takeda, S. Akane, T. Itou, A. Hoshiko, and K. En, "Role of boric acid for a poly ( vinyl alcohol ) fi $1 \mathrm{~m}$ as a cross-linking agent : Melting behaviors of the fi lms with boric acid," Polymer (Guildf)., vol. 51, no. 23, pp. 5539-5549, 2010.

[5] X. Bai, Z. Ye, Y. Li, L. Zhou, and L. Yang, "Preparation of crosslinked macroporous PVA foam carrier for immobilization of microorganisms," vol. 45, pp. 60-66, 2010.

[6] R. Parhi, "Cross-Linked Hydrogel for Pharmaceutical Applications : A Review," Tabriz Univ. Med. Sci., vol. 7, no. 4, pp. 515-530, 2017.

[7] N. Bhattarai, J. Gunn, and M. Zhang, "Chitosan-based hydrogels for controlled , localized drug delivery 认," Adv. Drug Deliv. Rev., vol. 62, no. 1, pp. 83-99, 2010.

[8] G. B. Mcguinness, "Polyvinvyl Alcohol-Based Cryogels : Tissue Engineering and Regenerative Medicine," pp. 1-12, 2014.

[9] M. H. El-Naas, A. H. I. Mourad, and R. Surkatti, "Evaluation of the characteristics of polyvinyl alcohol (PVA) as matrices for the immobilization of Pseudomonas putida," Int. Biodeterior. Biodegrad., vol. 85, pp. 413-420, 2013.

[10] J. Oh et al., "Development of polyvinyl alcohol - sodium alginate gel-matrix-based wound dressing system containing nitrofurazone," vol. 359, pp. 79-86, 2008.

[11] A. Joshi et al., "Functional compressive mechanics of a PVA / PVP nucleus pulposus replacement," vol. 27, pp. 176-184, 2006.

[12] S. R. Stauffer and N. A. Peppast, "Poly ( vinyl alcohol ) hydrogels prepared by freezing-thawing cyclic processing," vol. 33, no. 18, pp. 3932-3936, 1992.

[13] S. Jiang, S. Liu, and W. Feng, "PVA hydrogel properties for biomedical application," J. Mech. Behav. Biomed. Mater., vol. 4, no. 7, pp. 1228-1233, 2011.

[14] V. Pazos, R. Mongrain, and J. C. Tardif, "Polyvinyl alcohol cryogel : Optimizing the parameters of cryogenic treatment using hyperelastic models," J. Mech. Behav. Biomed. Mater., vol. 2, no. 5, pp. 542 549, 2009. 